\section{A promotory effect of inflammation on tumour initiation}

from A. J.S. Davies

To its devotees tumour immunology is an absorbing pastime. To them the facts that $T$ lymphocytes and B lymphocytes and macrophages can all in their different ways be cytotoxic, at least in vitro, are mutually compatible. The existence of anti-tumour antibodies that can be thought of as cytotoxic or enhancing or blocking is regarded with joy. The possibility that antibody-tumour-antigen complexes or tumour antigen alone can reduce the aggressive anti-tumour effects of some of the various kinds of hostile lymphocytes becomes part of the grand design. On this euphoric wave of complexity the tumour immunologists are carried as they invent even more and bizarre ways of immunotherapy. Their methodology comes increasingly to resemble some of the early attempts to contro bacterial diseases by methods that retrospectively were seen to be nonspecific and unreliable.

This is not to say that the tumour immunologists are wrong all the way along the line. There may really be an immunological surveillance mechanism which eradicates most tumours before they become a nuisance. When this mechanism has failed there may be tumour-specific immune responses in all instances which can be manipulated for the benefit of the patient. But the greater the economic pressure on scientists engaged in this biomedical research the more positive will be the assertions, not necessarily justified, that a particular theory is correct. A recent paper in the British Journal of Cancer by van den Brenk et al. (30, 246-260; 1974) blows a breath of fresh air into the arena.

Van den Brenk and his colleagues have worked on the effects of various inflammatory and anti-inflammatory agents on the survival and growth of clones of injected foreign tumour cells in rats. For example, they found that injection of cellulose sulphate ten minutes before intravenous injection of the Walker-256 adenocarcinoma caused substantial increases in the number of tumour cells starting to grow in the lungs and as a consequence a marked increase in tumour weight. This was even true in immune mice though immunisation always had some protective effect. From other experiments cellulose sulphate is known to be a powerful inducer of inflammation by some mechanism that is not altogether clear. Cellulose sulphate is also an anticoagulant and can be fibrinolytic.
In spite of the complexity of its effects the authors of the experiments feel and present additional evidence that the inflammatory properties of cellulose sulphate are those responsible for its augmentary effect on tumour seeding and thus tumour growth. In other experiments injection of a lung homogenate is shown to have a similar augmentary effect by means which are thought to be pharmacologically the same as those involved in the effect of cellulose sulphate. Direct effects of either lung homogenate or cellulose sulphate on tumour gnowth seem to be ruled out in control experiments. Experiments were performed in which tumour and inflammatory agents were injected in the rats' forepaws. Again cellulose sulphate caused some enhancement of tumour growth.

It would be nice to see these experiments repeated with syngeneic tumour implants. But there are many interesting corollaries of the present findings which should be read about in the original. One of the specific points made by the authors is worth underlining here. They say that a situation can arise wherein the survival and growth of tumour cells are inhibited by immunity but stimulated by inflammation resulting from the destruction of participating host and tumour cells. This provides food for thought for tumour immunotherapist and radiotherapist alike especially as Stjernswärd now claims to have evidence (Lancet, ii, 1285 ; 1974) for decreased survival related to postoperative radiation in early operable breast cancer. Van den Brenk and his associates have shown moreover that injection of an antiinflammatory steroid could reduce the augmentary effect of cellulose sulphate on the seeding of tumour cells. The old fashioned view that tumours should be thought of in the light of the naturenurture paradigm is perhaps worth reconsideration panticularly if damaged cells can nurture (albeit indirectly) their intact tissue-mates.

\section{Superfluid ${ }^{3} \mathrm{He}$ at zero pressure}

\section{from P. V. E. McClintock}

LiQuid ${ }^{3} \mathrm{He}$ has been cooled to temperatures below $0.7 \mathrm{mK}$ in a nuclear demagnetisation cryostat and, under its own saturated vapour pressure, it was found to undergo a superfluid transition at $0.93 \mathrm{mK}$. The experiment, which was performed at the Helsinki University of Technology by Ahonen, Haikala, Krusius and Lounasmaa, is described in a recent issue of Physical Review Letters $(33,628 ; 1974)$.

Since the discovery in 1972 that liquid ${ }^{3} \mathrm{He}$ under a pressure of 24 atmospheres undergoes two separate transitions at temperatures of about 2.7 and $2.2 \mathrm{~K}$, intensive efforts, both theoretical and experimental, have been made towards gaining an understanding of the two new phases of the liquid. The experiments have, until now, all employed one of two cooling methods: either compressional solidification of ${ }^{3} \mathrm{He}$, known as Pomeranchuk cooling, which restricts experiments to the solidification pressure of 34 atmospheres; or adiabatic demagnetisation of cerium magnesium nitrate (CMN), which can be carried out at any pressure. Using these techniques, a remarkable amount of information concerning the nature of the new phases has been gained from a variety of experiments including, in particular, nuclear magnetic resonance (NMR) measurements. It has been shown that both the higher temperature so-called $\mathrm{A}$ phase and the lower temperature B phase exhibit superfluid properties; and that, as the pressure is reduced with zero applied magnetic field, the higher temperature transition moves to lower temperatures, while the lower temperature one moves to higher temperatures, with the two transitions meeting in a tricritical point at about 20 atmospheres. Below 20 atmospheres only the B phase is stable, and the transition was found to move to lower temperatures with falling pressure. It was not feasible by demagnetising $\mathrm{CMN}$ to follow the transition below about 15 atmospheres and $2.2 \mathrm{mK}$, so that is was impossible to say whether or not the transition would ever reach zero pressure: it seemed quite credible that ${ }^{3} \mathrm{He}$ might not be superfluid under its saturated vapour pressure, but would always require the application of external pressure to undergo the superfluid transition.

The ultimate low temperature attainable with $\mathrm{CMN}$ is related to the spontaneous ordering of the electron spins on the cerium ions as a result of their mutual magnetic interaction. To reach temperatures significantly below $2 \mathrm{mK}$ an assembly whose magnetic elements interact less strongly is therefore required, and the Helsinki group chose to demagnetise copper nuclei in order to achieve their cooling effect. In essence, the technique is simple. When a large magnetic field is applied at a starting temperature of less than $20 \mathrm{mK}$, the copper nuclei tend to align themselves, and heat is liberated and is removed by means of a dilution refrigerator. The thermal link to the refrigerator is then broken and the magnetic field very slowly reduced to zero, resulting in cooling as the nuclei disorientate themselves again.

In practice, great care has to be taken in minimising heating due to the 